\title{
Satisfacción laboral y compromiso organizacional de docentes en una universidad pública de Perú
}

\section{Job satisfaction and organizational commitment of teachers in a public university in Peru}

\author{
Ronald Quispe Flores ${ }^{1}$, Soledad Paucar Sullca ${ }^{2}$ \\ Universidad César Vallejo, Trujillo, Perú ${ }^{1}$. \\ Universidad Nacional Amazónica de Madre de Dios, Puerto Maldonado, Perú1. \\ Universidad Nacional Amazónica de Madre de Dios, Puerto Maldonado, Perú .
}

Orcid ID: https://orcid.org/0000-0002-2212-267X ${ }^{1}$

Orcid ID: https://orcid.org/0000-0002-7374-7776

Recibido: 10 de julio de 2019

Aceptado 07 de diciembre de 2019

\section{Resumen}

El objetivo de esta investigación fue evaluar la relación entre satisfacción laboral y compromiso organizacional de docentes de una universidad pública en el Perú, utilizando el diseño explicativo secuencial (QUAN $\rightarrow$ qual) del enfoque mixto. La población de estudio consistió en 256 docentes. El análisis cuantitativo de encuestas obtenidas de 114 docentes elegidos mediante el muestreo intencional, se realizó mediante estadísticas descriptivas y análisis de regresión logística multivariada. El análisis cualitativo de entrevistas obtenidas de 8 docentes elegidos de manera propositiva, se realizó mediante el análisis etnográfico. Los resultados indicaron que: los docentes satisfechos laboralmente tienen 3.39 veces mayor la posibilidad de tener compromiso organizacional, comparado con los docentes insatisfechos $(\widehat{O R}=$ 3.39, IC 95\%: $1.22-9.44)$; el 48.3\% tienen insatisfacción laboral y el 70.2\% no tienen compromiso de continuidad; las principales razones fueron bajas remuneraciones, falta promoción y nombramiento, inconformidad con el reconocimiento y escasas oportunidades de crecimiento. Sin embargo, el $82.5 \%$ tienen compromiso afectivo y el $57 \%$ compromiso normativo, como reconocimiento a la oportunidad laboral que encontraron en la universidad. En conclusión, existe relación directa significativa entre la satisfacción laboral y el compromiso organizacional; el descuido de los factores higiénicos y motivacionales, ocasionan la insatisfacción laboral y falta de compromiso de continuidad.

${ }^{1}$ Correspondencia al autor

E-mail: rquispe@unamad.edu.pe 
Palabras clave: Satisfacción laboral, compromiso organizacional, investigación de métodos mixtos, análisis de regresión múltiple, universidad, docencia.

\begin{abstract}
The objective of this research was to evaluate the relationship between job satisfaction and organizational commitment of teachers from a public university in Peru, using the sequential explanatory design (QUAN $\rightarrow$ qual) of the mixed approach. The study population consisted of 256 teachers. The quantitative analysis of surveys obtained from 114 teachers chosen through intentional sampling was performed using descriptive statistics and multivariate logistic regression analysis. The qualitative analysis of interviews obtained from 8 teachers chosen on a proactive basis was carried out through ethnographic analysis. The results indicated that: satisfied teachers have 3.39 times greater the possibility of having an organizational commitment, compared to dissatisfied teachers $((\mathrm{OR})=3.39,95 \% \mathrm{CI}: 1.22-9.44) ; 48.3 \%$ have dissatisfaction at work and $70.2 \%$ no have commitment to continuity; the main reasons were low salaries, lack of promotion, lack of appointment, lack of recognition and opportunities for growth. However, in recognition of the job opportunity they found at the university, $82.5 \%$ have an affective commitment and 57\% have a normative commitment. In conclusion, there is a significant direct relationship between job satisfaction and organizational commitment; the neglect of the hygienic and motivational factors, cause job dissatisfaction and lack of commitment to continuity.
\end{abstract}

Keywords: Job satisfaction, organizational commitment, mixed methods research, multiple regression analysis, university, teaching.

\title{
Introducción
}

La satisfacción laboral (SL) y el compromiso organizacional (CO) son las actitudes más importantes relacionadas con el trabajo y a la vez, constituyen los problemas cruciales para las organizaciones. En estas, los empleados satisfechos están comprometidos con sus organizaciones, por lo que muestran una actitud positiva hacia su trabajo e incluso superan las expectativas normales (Robbins \& Coulter, Management, 2012). El CO de los profesores es un factor crítico para la mejora del proceso enseñanza-aprendizaje $\mathrm{y}$, por consecuencia, para incrementar la calidad de la educación (Maldonado-Radillo, Barón, Rivera, \& Venegas, 2014).

Por estas razones, la relación entre la SL y CO de los profesores de educación secundaria a nivel nacional y mundial, fue ampliamente estudiada (Tentama \& Pranungsari, 2016; Getahun, Tefera, \& Burichew, 2016; Werang \& Agung, 2017); y en todos ellos, se ha logrado determinar que la SL, tiene correlación positiva significativa con el CO; es decir, cuanto mayor sea la SL del docente, mayor será su CO en la institución educativa. 
Respecto a la SL y CO de los trabajadores (personal académico y administrativo) de institutos tecnológicos superiores, también se encontró una correlación positiva y significativa (Nieto, 2017; Ramos \& Santa Cruz, 2018). Sin embargo, a nivel universitario-y más aun en el sistema universitario público--se han encontrado pocas investigaciones sobre SL y CO de docentes universitarios. Tras la revisión de los mismos (Hurtado, 2014; Poornachandrika, 2015; Luna, 2015; Mena, 2016; Muhammad Hammad Ali \& Imran Bashir, 2018; Valdivia, 2018), tambien se ha podido encontrar que, la relación de estas variables es al menos moderada, directa y significativa.

Casi en la totalidad de los trabajos revisados, el análisis de relación o correlación de variables de interés, se hizo mediante el análisis de regresión lineal simple, el análisis de correlación de Pearson o el coeficiente de correlación de Spearman. Es así que, en la revisión sistemática de las tendencias del diseño metodológico usadas en los artículos de investigación cuya temática principal fue la SL del profesorado universitario, el 29.2\% utilizó el análisis de regresión lineal, para el análisis inferencial (Álvarez, 2019).

Sin embargo, es preciso indicar que el coeficiente de correlacíon es una medida de correlación lineal entre dos variables cuantitativas con distribuciones normales. De la misma forma, para el análisis de regresión lineal simple, existe una serie de supuestos que se deben cumplir para la variable respuesta cuantitava (Casella \& Berger, 1990). Por otra parte, la inferencia de asociación de variables utilizando el coeficiente de correlación de Spearman, sólo se puede realizar si las variables se encuentran al menos en escala ordinal y los sujetos para el estudio hayan sido elegidos aleatoriamente (Siegel \& Castellan, 1998).

La SL y el CO son dos constructos (variables no directamente medibles, menos directamente cuantificables) (Álvarez, 2019; Maldonado-Radillo, Barón, Rivera, \& Venegas, 2014) para los cuales, la aplicación de el análisis de correlación, análisis de regresión lineal simple o la determinación del coeficiente de correlación de Spearman, pueden conducir a sesgos en los resultados.

También se observó que, en la mayoría de las investigaciones, los datos se recolectaron en un corto periodo de tiempo (diseño transversal) a profesores que de forma voluntaria decidieron participar en el estudio (muestreo no probabilístico); ello implica que no se tuvo control sobre las posibles variables confusoras, que tambien pueden constituirse en fuente de sesgo para los resultados. Al respecto, para evitar este problema (Henquin, 2013), a modo de estrategia se sugiere el uso de análisis multivariante. 
Liu \& Bellibas (2018), indican que la SL y el CO de los directores de colegios, según países y continentes presentan diferencias significativas; asimismo, manifiestan que los factores influyentes en la SL y CO de los directores, generalmente no son consistentes en los distintos contextos.

Bajo estas consideraciones, el objetivo de la investigación fue conocer la SL y el CO de los docentes de la Universidad Nacional Amazónica de Madre de Dios, asimismo, evaluar la relación entre estas variables, mediante el enfoque de investigación mixta (QUAN $\rightarrow$ qual); donde, en la fase cuantitativa, el análisis de datos se realizó mediante la regresión logística multivariada. Luego, a fin de entender de mejor forma los resultados de esta fase, se realizó la investigación cualitativa utilizando el método de investigación fenomenológico.

\section{Metodología}

\section{Diseño}

El diseño de investigación utilizado fue el diseño explicativo secuencial (QUAN $\rightarrow$ qual) del enfoque de investigación mixta (cuantitativo cualitativo).

Según Creswell (2014), el diseño de investigación se denomina explicativo porque los resultados de los datos cuantitativos de la primera fase, son explicados con los resultados de los datos cualitativos de la segunda fase. Es considerado secuencial, porque la fase inicial cuantitativa será seguida por la fase cualitativa.

En la fase cuantitativa, el diseño de investigación utilizado fue el diseño transversal observacional, dado que los datos fueron recogidos en un corto periodo de tiempo y ninguna variable fue manipulada, tampoco las unidades de estudio fueron asignados a ningún grupo experimental. El método de investigación aplicada fue el método analítico-sintético, porque la hipótesis planteada para la variables en estudio, fue probada mediante hipótesis espécificas. Respecto al método analítico-sintético, Rodríguez \& Pérez (2017) indican que:

(...) se refiere a dos procesos intelectuales inversos que operan en unidad: el análisis y la síntesis. El análisis es un procedimiento lógico que posibilita descomponer mentalmente un todo en sus partes y cualidades, en sus múltiples relaciones, propiedades y componentes. Permite estudiar el comportamiento de cada parte. La síntesis es la operación inversa, que establece mentalmente la unión o combinación de las partes previamente analizadas y posibilita descubrir relaciones y características generales entre los elementos de la realidad (...) (p.186). 
En la fase cualitativa, se utilizó el método de investigación fenomenológico, dado que se procuró conocer aspectos subjetivos de los participantes respecto a las variables de estudio. Respecto a este método, Vargas (2011) manifiesta que:

(...) es un método más adecuado para abordar investigaciones que tienen que ver con el mundo interior de las personas. Se centra en la interpretación de los "fenómenos" tal y como se le presentan a la persona en su fuero interno. Para este método, la realidad no está fuera de manera objetiva, sino que se constituye subjetivamente de aquello que sucede en el interior de las personas como consecuencia del vivir y al margen de toda teoría (...) (p.33,34).

\section{Participantes}

La población de estudio estuvo conformada por 269 docentes-entre contratados y nombrados_-, quienes se encontraban laborando en la Universidad Nacional Amazónica de Madre de Dios. En la fase cuantitativa, precisamente en el ajuste del modelo de regresión logística a los datos, se estimaron 10 coeficientes correspondientes al mismo número de variables artificiales (dummy) generadas a partir de las siete variables explicativas; por lo que, según Long \& Jeremy Freese (2001), el tamaño mínimo de muestra requerido es 100. Sin embargo, de manera voluntaria aceptaron participar 114 docentes, constituyendo ellos la muestra de estudio. En la fase cualitativa se eligieron de manera propositiva 8 docentes, a razón de la saturación de las categorías de análisis.

\section{Instrumentos}

El cuestionario utilizado para medir la SL fue la adaptación del cuestionario utilizado por Dingeta (2013) para este mismo propósito, que a su vez fue acondicionado del instrumento original elaborado y validado por Al-Rubaish, et al. (2011). Este constaba de 9 dimensiones o “facetas": reconocimiento, pago, promoción y oportunidades de crecimiento, relación con los compañeros de trabajo, supervisión, entorno físico y comodidades, autonomía, trabajo en sí y carga de trabajo; con un total de 27 indicadores, distribuidos en las 9 dimensiones. Su validez respecto a su forma, contenido y estructura fue evaluado por 3 expertos. La confiabilidad fue evaluada mediante el coeficiente alfa de Cronbach, cuyo resultado fue 0.850 , el cual indica que el instrumento es confiable (Barraza Macías, 2007).

Para medir el CO, se adaptó el cuestionario utilizado por Dingeta (2013) y está basado en la escala de CO de Meyer y Allen (Meyer \& Allen, 1997). Consta de 3 dimensiones: 
compromiso afectivo, de continuidad y normativo. Tiene 13 indicadores distribuidos en las 3 dimensiones. La validez respecto a su forma, contenido y estructura fue evaluado por 3 expertos. La confiabilidad fue evaluada mediante el coeficiente alfa de Cronbach, cuyo resultado fue 0.759, lo cual indica que el instrumento es confiable (Barraza Macías, 2007). En ambos instrumentos, las respuestas fueron puntuaciones en escala Likert.

Para la fase cualitativa, se utilizó una guía de entrevista semi-estructurada elaborada según los resultados de la fase cuantitativa. Respecto a su validez, Alvarez-Gayou Jurgenson (2003), indica que éste es un postulado del enfoque cuantitativo y una forma de evaluarla es, por ejemplo, mediante la triangulación de métodos o de investigadores. Si bien esta práctica enriquece la investigación, no se considera indispensable. En este entender, es más preferible la autenticidad de los resultados que la validez; es decir, que las personas logren comunicar su sentir real. Respecto a su confiabilidad, el mismo autor manifiesta que, en el enfoque cuantitativo, ésta busca reducir al mínimo el error de medición; sin embargo, en el enfoque cualitativo, no se realizan mediciones, por lo que la posibilidad de determinación de la fiabilidad queda anulada.

\section{Recolección de datos}

En la fase cuantitativa, los datos para la SL y el CO se recolectaron entre octubre y noviembre del año 2018, mediante encuestas autoadministradas a 114 docentes elegidos mediante muestreo intencional. En la fase cualitativa, los datos se recopilaron mediante entrevistas a los docentes elegidos de manera propositiva en diciembre del 2018 y enero del 2019.

\section{Análisis de datos}

Según las puntuaciones en escala Likert, a cada docente se le calculó la puntuación promedio para cada ítem de SL, de tal forma que un docente con puntuación promedio menor a tres, se consideró "dissatisfecho"2; con promedio igual a tres "indiferente"; y con puntuación promedio superior a tres, "satisfecho" (Dingeta, 2013). Este procedimiento se siguió para cada dimensión de SL, como también para la SL general. Por otro lado, para describir las variables en estudio, se elaboraron cuadros de distribución de frecuencias y gráficos estadísticos, con el apoyo del programa de hoja de cálculo para computadora Microsoft Excel y el software estadístico SPSS, versión 22.

${ }^{2}$ La dissatifacción laboral, hace referencia a una baja o muy baja satisfacción laboral. 
En cuanto al $\mathrm{CO}$ y sus tres dimensiones, se procedió de manera similar que para la SL. Un docente con puntuación promedio menor a tres, se consideró "sin compromiso"; con puntuación promedio igual a tres "indiferente"; y con una puntuación promedio superior a tres "comprometido" (Dingeta, 2013).

La relación de la SL con las dimensiones del CO—el CO general controlando las variables sociodemográficas - se evaluó calculando intervalos de confianza para la razón de posibilidades (odds ratio: OR), mediante el ajuste del modelo de regresión logística multivariada a los datos. Cuando el intervalo de confianza calculado para la razón de posibilidades no contenía a 1, a un nivel de confianza del 95\%, se concluyó que existía una relación estadísticamente significativa entre las variables; caso contrario, se concluyó que no existía relación significativa. En este caso fue necesario utilizar el software estadístico STATA, versión 14.

En la fase cualitativa, las grabaciones de las entrevistas fueron transcritas a texto. Posteriormente, mediante la categorización, ${ }^{3}$ realizar los "análisis etnográficos (puramente descriptivos, tratando de dar una imagen fiel a la realidad)" (Monje Álvarez, 2011, p. 192). Cabe precisar que las categorías, como es usual en entrevistas semi estructuradas, se definieron antes de la ejecución, a partir de resultados obtenidos en la fase cuantitativa.

\section{Resultados}

En la tabla 1, se observa que el $49.1 \%$ de los docentes participantes en el estudio, se encontraban satisfechos. Sólo el 2.6\% se encontraban indiferentes; es decir, no estaban seguros de su dissatisfacción o satisfacción laboral. También se puede observar que aproximadamente la mitad de los docentes encuestados (48.3\%), se encontraban dissatisfechos.

Tabla 1

Satisfacción laboral de los docentes de una universidad pública del Perú

\begin{tabular}{lccc}
\hline Nivel de satisfacción & Cantidad & Porcentaje (\%) & Porcentaje acumulado (\%) \\
\hline Dissatisfecho & 55 & 48.3 & 48.3 \\
Indiferente & 3 & 2.6 & 50.9 \\
Satisfecho & 56 & 49.1 & 100.0 \\
Total & 114 & 100.0 & \\
\hline
\end{tabular}

${ }^{3}$ La categorización consiste en la segmentación en elementos singulares, o unidades que resultan relevantes y significativas desde el punto de vista de nuestro interés investigativo (Monje Álvarez, 2011, p. 194). 
Elaboración: Propia

Dado que la dissatisfacción puede tener efectos negativos en el desempeño laboral de los docentes, es necesario conocer con exactitud, que facetas o dimensiones de la SL frecuentemente presentan este problema.

Precisamente, en la tabla 2 y figura 1, se observa que los docentes en mayor porcentaje se encontraban dissatisfechos con el pago $(73.7 \%)$, promoción y oportunidades de crecimiento (72.8\%) y el reconocimiento (72\%). Asimismo, el 48.2\% se encontraban dissatisfechos con el entorno físico y comodidades, el $39.5 \%$ con la relación con compañeros de trabajo. Sin embargo, el $96.5 \%$ se encontraban satisfechos con el trabajo en sí que realizan, el $73.7 \%$ con la autonomía, el $62.3 \%$ con la carga de trabajo, y el $60.5 \%$ con la supervisión.

Tabla 2

Nivel de satisfacción laboral de los docentes, según dimensión

\begin{tabular}{lccc}
\hline \multirow{2}{*}{ Dimensión } & \multicolumn{3}{c}{ Nivel de satisfacción (\%) } \\
\cline { 2 - 4 } & Dissatisfecho & Indiferente & Satisfecho \\
\hline Reconocimiento & 72.0 & 14.0 & 14.0 \\
Pago & 73.7 & 7.9 & 18.4 \\
Promoción y oportunidades de crecimiento & 72.8 & 7.0 & 20.2 \\
Relación con los compañeros de trabajo & 39.5 & 8.8 & 51.7 \\
Supervisión & 33.4 & 6.1 & 60.5 \\
Entorno físico y comodidades & 48.2 & 23.7 & 28.1 \\
Autonomía & 9.6 & 16.7 & 73.7 \\
Trabajo en sí & 2.6 & 0.9 & 96.5 \\
Carga de trabajo & 23.7 & 14.0 & 62.3 \\
\hline
\end{tabular}

Elaboración: Propia 


\section{Figura 1}

Nivel de satisfacción laboral de los docentes, según dimensión

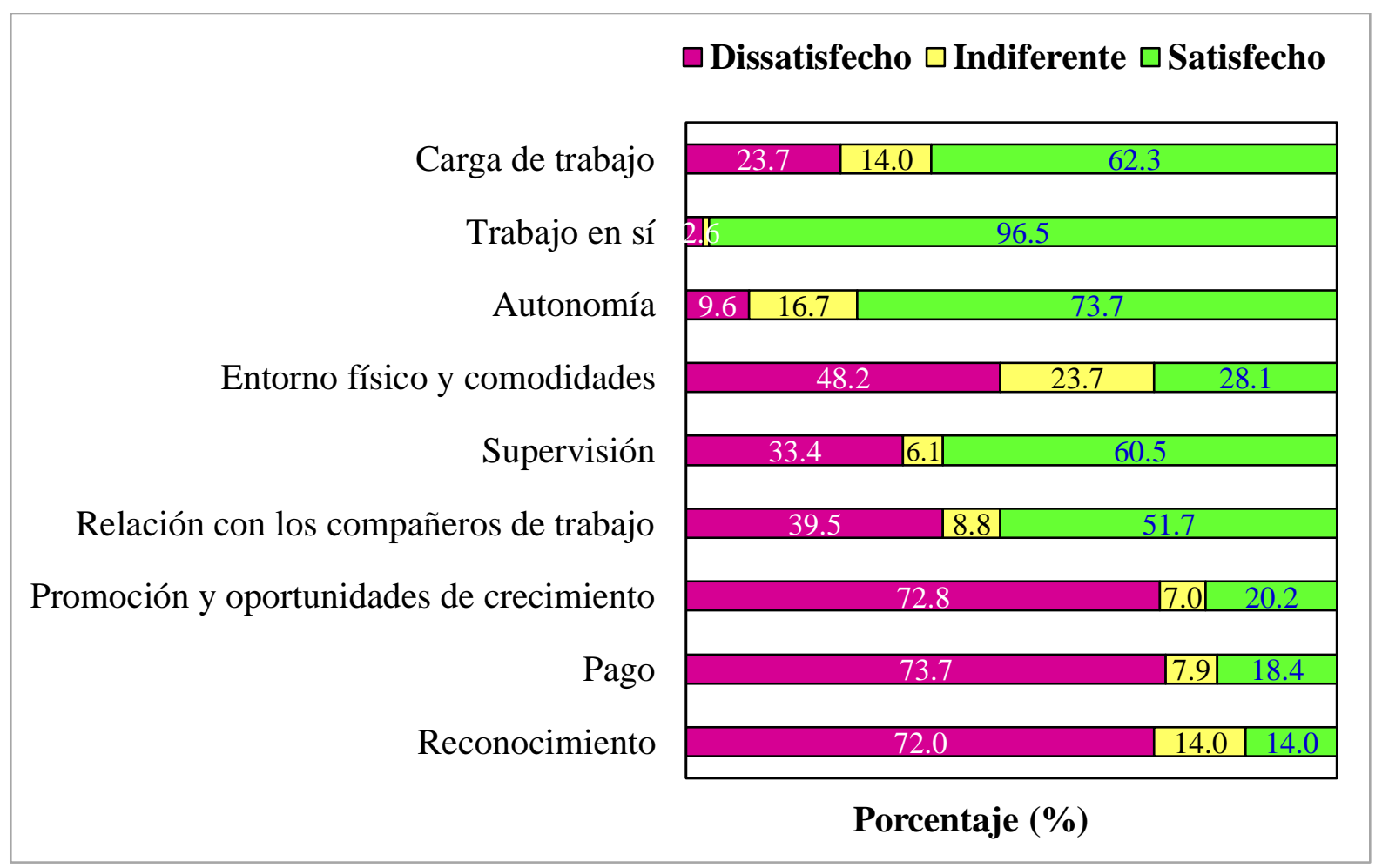

Fuente: Elaboración propia

Respecto al CO, como se observa en la tabla 3, el $75 \%$ de los docentes se encontraban comprometidos con el cumplimiento de los fines y objetivos de la universidad; lo contrario ocurría con los restantes $25 \%$. No se observó ningún docente indiferente con su compromiso, es decir, el CO se comportó como una variable dicotómica.

Tabla 3

Compromiso organizacional de los docentes

\begin{tabular}{lcc}
\hline Nivel de compromiso & Cantidad & Porcentaje (\%) \\
\hline No comprometido & 29 & 25 \\
Comprometido & 85 & 75 \\
Total & 114 & 100 \\
\hline Fuente: Elaboración propia & &
\end{tabular}

Fuente: Elaboración propia

Aún en bajo porcentaje la falta de compromiso llama a la reflexión, por ello es necesario realizar el análisis del CO según sus dimensiones. 
Es así que en la tabla 4 y la figura 2, se observa que el $70.2 \%$ de docentes no se encontraban decididos de continuar en la universidad, sin embargo, el $82.5 \%$ tenían compromiso afectivo. Asimismo, el 57\% indicaron tener compromiso normativo.

Tabla 4

Compromiso organizacional de los docentes, según dimensiones.

\begin{tabular}{lcc}
\hline \multirow{2}{*}{ Dimensión } & \multicolumn{2}{c}{ Nivel de compromiso (\%) } \\
\cline { 2 - 3 } & No comprometido & Comprometido \\
\hline Afectivo & 17.5 & 82.5 \\
De continuidad & 70.2 & 29.8 \\
Normativo & 43.0 & 57.0 \\
\hline Elaboración: Propia & &
\end{tabular}

Figura 2

Compromiso organizacional de los docentes, según dimensiones

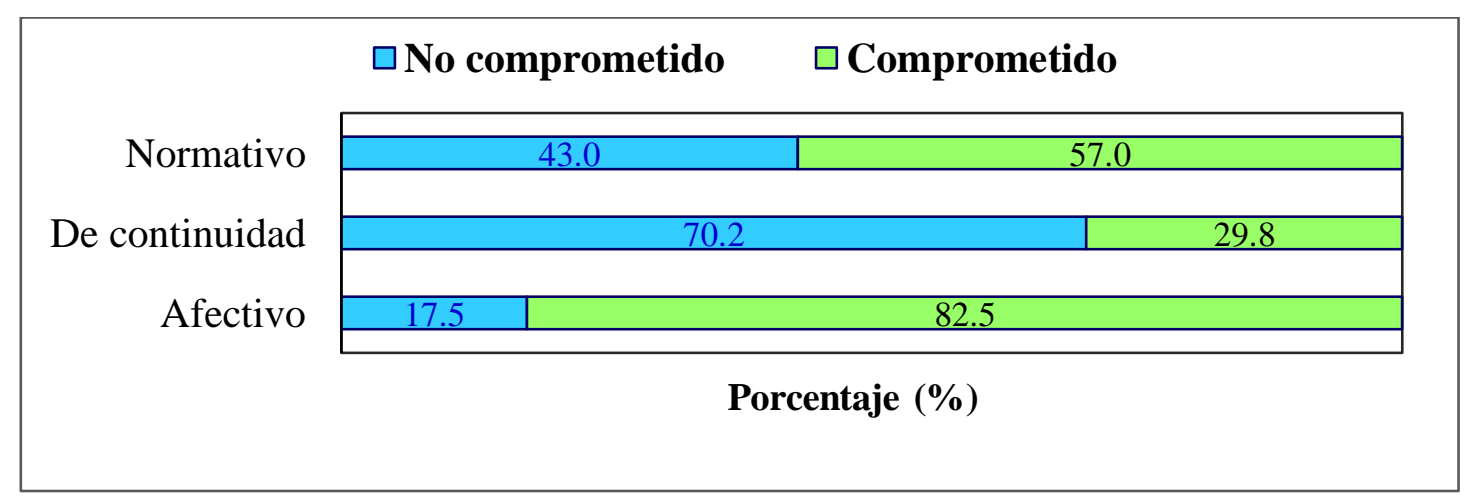

Fuente: Elaboración propia

Por otro lado, a fin de evitar el efecto de posibles variables confusoras (condición laboral, grado académico, experiencia laboral edad y sexo del docente) en la evaluación de la relación entre la variables de interés, se estimó la razón de posibilidades mediante el ajuste del modelo de regresión logística multivariada a los datos (Henquin, 2013).

Evaluando la bondad de ajuste del modelo, se determina que, al menos una de las variables consideradas en el modelo, explica de manera significativa la variabilidad del compromiso afectivo (p-valor < 0.05) (véase tabla 5). Precisamente esa variable viene a ser la SL, dado, que el intervalo de confianza estimado para la asociación entre la SL y compromiso afectivo OR, al nivel de confianza de1 95\%, (I.C.(95\%); 1.35 - 16.83), no contiene a 1. Es decir, 
existe una relación estadísticamente significativa entre SL y el compromiso afectivo de los docentes.

En la tabla 5, también se observa que el $O R$ ajustado fue $\widehat{O R}=4.76$, ello indica que, los docentes satisfechos laboralmente tienen 4.76 veces mayor la posibilidad de tener compromiso afectivo con la universidad, comparado con los docentes dissatisfechos; manteniendo constantes las otras variables explicativas incluidas en el modelo.

\section{Tabla 5}

Variables relacionadas con el compromiso afectivo

\begin{tabular}{|c|c|c|}
\hline \multirow{2}{*}{ Variable } & \multirow{2}{*}{$\widehat{O R}$} & I.C. 95\% \\
\hline & & L.i. \\
\hline
\end{tabular}

\section{Satisfacción laboral}

Indiferente

1.00

Satisfecho

4.76

1.35

16.83

\section{Condición laboral}

Nombrado

$0.11 \quad 0.01$

0.91

\section{Grado académico}

Magister

$\begin{array}{lll}2.58 & 0.54 & 12.27\end{array}$

Doctor

$1.59 \quad 0.24$

10.35

\section{Experiencia laboral (años)}

De 3 a menos de 5

$\begin{array}{lll}4.93 & 0.38 & 64.72 \\ 2.35 & 0.35 & 16.00 \\ 1.87 & 0.20 & 17.84\end{array}$

De 5 a menos de 10

17.84

\section{Edad (años)}

De 40 a 49

$\begin{array}{lll}3.24 & 0.53 & 19.65\end{array}$

De 50 a más

$2.73 \quad 0.44$

16.87

Sexo

\begin{tabular}{lccc} 
Masculino & 2.10 & 0.61 & 7.28 \\
Constante & 0.68 & 0.09 & 5.28 \\
\hline Estadístico calculado: LR chi2(10) $=21.68$; p-valor $=0.0168$. \\
Categorías de referencia: Dissatisfecho, contratado, Bachiller, Menos \\
de 3 años, Menos de 40 años, Femenino. \\
Elaboración: Propia.
\end{tabular}

En la tabla 6, se observa que, a un nivel de confianza del 95\%, el modelo ajustado no es significativo ( $\mathrm{p}$-valor $\geq 0.05$ ). Por lo tanto, no existe relación significativa entre la SL y el 
compromiso de continuidad de los docentes, ello queda confirmado por el intervalo de confianza calculado para la razón de posibilidades OR correspondiente a la SL (I.C.(95\%): 0.44 $-3.15)$ que contiene a 1.

Tabla 6

Variables relacionadas con el compromiso de continuidad

\begin{tabular}{|c|c|c|c|}
\hline \multirow{2}{*}{ Variable } & \multirow{2}{*}{$\widehat{O R}$} & \multicolumn{2}{|c|}{ I.C. 95\% } \\
\hline & & L.i. & L.S. \\
\hline \multicolumn{4}{|c|}{ Satisfacción laboral } \\
\hline Indiferente & 1.00 & & \\
\hline Satisfecho & 1.18 & 0.44 & 3.15 \\
\hline
\end{tabular}

\section{Condición laboral}

Nombrado

$0.46 \quad 0.10 \quad 2.06$

\section{Grado académico}

$\begin{array}{llll}\text { Magister } & 4.77 & 1.16 & 16.61 \\ \text { Doctor } & 5.75 & 1.10 & 30.07\end{array}$

\section{Experiencia laboral (años)}

De 3 a menos de 5

$0.27 \quad 0.05 \quad 1.39$

De 5 a menos de 10

$\begin{array}{lll}0.20 & 0.04 & 0.87\end{array}$

De 10 a más

$0.53 \quad 0.11$

\section{Edad (años)}

De 40 a 49

$\begin{array}{lll}1.40 & 0.39 & 5.01\end{array}$

De 50 a más

$3.69 \quad 0.93$

Sexo

\begin{tabular}{lccc} 
Masculino & 0.65 & 0.22 & 1.93 \\
Constante & 0.25 & 0.04 & 1.60 \\
\hline Estadístico calculado: LR chi2(10) $=17.87$; p-valor $=0.0572$. \\
Categorías de referencia: Dissatisfecho, contratado, Bachiller, Menos \\
de 3 años, Menos de 40 años, Femenino. \\
Elaboración: Propia.
\end{tabular}

Se observa en la tabla 7, que el ajuste del modelo para evaluar la relación entre la SL y el compromiso normativo resulta ser no significa ( $\mathrm{p}$-valor $\geq 0.05$ ), lo cual indica que no existe relación entre SL y el compromiso normativo.

\section{Tabla 7}


Relación entre satisfacción laboral y el compromiso normativo

\begin{tabular}{|c|c|c|c|}
\hline \multirow{2}{*}{ Variable } & \multirow{2}{*}{$\widehat{O R}$} & \multicolumn{2}{|c|}{ I.C.95\% } \\
\hline & & L.i. & L.s. \\
\hline \multicolumn{4}{|l|}{ Satisfacción laboral } \\
\hline Indiferente & 1.00 & & \\
\hline Satisfecho & 1.56 & 0.66 & 3.72 \\
\hline \multicolumn{4}{|l|}{ Condición laboral } \\
\hline Nombrado & 1.09 & 0.30 & 3.94 \\
\hline \multicolumn{4}{|l|}{ Grado académico } \\
\hline Magister & 0.76 & 0.27 & 2.16 \\
\hline Doctor & 0.75 & 0.20 & 2.81 \\
\hline \multicolumn{4}{|c|}{ Experiencia laboral (años) } \\
\hline De 3 a menos de 5 & 1.17 & 0.29 & 4.74 \\
\hline De 5 a menos de 10 & 1.61 & 0.45 & 5.80 \\
\hline De 10 a más & 0.41 & 0.10 & 1.71 \\
\hline \multicolumn{4}{|l|}{ Edad (años) } \\
\hline De 40 a 49 & 0.63 & 0.22 & 1.84 \\
\hline De 50 a más & 0.96 & 0.29 & 3.20 \\
\hline \multicolumn{4}{|l|}{ Sexo } \\
\hline Masculino & 1.30 & 0.50 & 3.39 \\
\hline Constante & 0.25 & 0.04 & 1.60 \\
\hline
\end{tabular}

Estadístico calculado: $\mathrm{LR}$ chi2(10) $=13.85 ; \mathrm{p}$-valor $=0.1802$

Categorías de referencia: Dissatisfecho, contratado, Bachiller, Menos de 3 años, Menos de 40 años, Femenino.

Elaboración: Propia.

El modelo ajustado a los datos para evaluar la relación entre la SL general y el CO general, resulta significativo ( $\mathrm{p}$-valor < 0.05) (véase tabla 8). Este resultado indica que al menos una variable explicativa considerada en el modelo de regresión logística multivariado, explica significativamente la variación en el CO de los docentes.

En la tabla 8, se observa que la SL tiene relación significativa con el CO, dado que el intervalo de confianza estimado para la razón de posibilidades $O R$ a nivel de confianza del 95\% (I.C.(95\%); 1.22 - 9.44), no contiene a 1. Asimismo, la razón de posibilidades $O R$ estimada resulta $\widehat{O R}=3.39$, lo cual indica que, los docentes satisfechos laboralmente, tienen 3,39 veces mayor la posibilidad de asumir CO con la universidad donde laboran, comparado con los 
docentes dissatisfechos; manteniendo controlado las otras variables explicativas incluidas en el análisis.

\section{Tabla 8}

Relación entre satisfacción laboral y el compromiso organizacional

\begin{tabular}{|c|c|c|c|}
\hline \multirow{2}{*}{ Variable } & \multirow{2}{*}{$\widehat{O R}$} & \multicolumn{2}{|c|}{ I.C. 95\% } \\
\hline & & L.i. & L.S. \\
\hline \multicolumn{4}{|l|}{ Satisfacción laboral } \\
\hline Indiferente & 1.00 & & \\
\hline Satisfecho & 3.39 & 1.22 & 9.44 \\
\hline \multicolumn{4}{|l|}{ Condición laboral } \\
\hline Nombrado & 0.39 & 0.08 & 2.06 \\
\hline \multicolumn{4}{|l|}{ Grado académico } \\
\hline Magister & 1.30 & 0.37 & 4.65 \\
\hline Doctor & 1.70 & 0.33 & 8.65 \\
\hline \multicolumn{4}{|c|}{ Experiencia laboral (años) } \\
\hline De 3 a menos de 5 & 0.62 & 0.12 & 3.07 \\
\hline De 5 a menos de 10 & 3.56 & 0.59 & 21.49 \\
\hline De 10 a más & 0.82 & 0.12 & 5.38 \\
\hline
\end{tabular}

Edad (años)

$\begin{array}{llll}\text { De } 40 \text { a } 49 & 1.16 & 0.31 & 4.42 \\ \text { De } 50 \text { a más } & 2.27 & 0.47 & 11.02\end{array}$

Sexo

\begin{tabular}{llll} 
Masculino & 2.09 & 0.73 & 6.00 \\
Constante & 0.88 & 0.15 & 5.24 \\
\hline
\end{tabular}

Estadístico calculado: $\mathrm{LR}$ chi2 $(10)=19.81 ; \mathrm{p}$-valor $=0.0311$.

Categorías de referencia: Dissatisfecho, contratado, Bachiller, Menos de 3 años, Menos de 40 años, Femenino.

Elaboración: Propia.

Para entender y explicar de mejor forma los resultados obtenidos en la etapa cuantitativa, a continuación, se presentan los resultados de la fase cualitativa, obtenidos mediante el análisis etnográfico:

\section{Pago}

Respecto a la disstatisfacción con el pago o remuneraciones, los docentes manifiestan que el sueldo que perciben es muy bajo comparado con el costo de la canasta familiar en la región. 
Si bien el sueldo lo fija el estado a través del Ministerio de Economía y Finanzas (MEF), las autoridades universitarias no hacen gestión para incrementar las remuneraciones a través de los recursos directamente recaudados $\mathrm{u}$ otras fuentes de financiamiento.

\section{Promoción y oportunidades de crecimiento}

Los docentes contratados manifiestan que ya mucho tiempo vienen esperando su nombramiento. Por su parte, los docentes nombrados manifiestan que hace buen tiempo vienen esperando su promoción a una categoría superior. Consideran hasta cierto punto esta situación como una señal de egoísmo por parte de las autoridades, para no realizar gestiones para los nombramientos y promociones.

\section{Reconocimiento}

Se considera que no hay una correspondencia entre el esfuerzo que realizan los docentes para especializarse y la remuneración que perciben. Si se dan reconocimientos, no siempre es para quienes lo merecen.

\section{Entorno físico y comodidades}

Respecto a este problema, se manifiesta que existe carencia en el equipamiento de las salas de docentes acorde a sus necesidades laborales. Las aulas y laboratorios no están debidamente equipados e implementados, si bien es cierto, en algunos casos están en proceso de ello.

\section{Relación con los compañeros de trabajo}

La dissatisfacción que surge en la relación con los compañeros de trabajo, es que cada docente busca superarse de una u otra forma y eso no necesariamente es bien vista por sus colegas. Respecto al alto porcentaje de docentes que no tienen el compromiso de continuar en la universidad (como se observa en la tabla 4 y figura 2), los docentes manifiestan que, en la universidad, no se sienten reconocidos más que todo económicamente, además de que las condiciones de vida en la zona de trabajo no son óptimas.

\section{Discusión}

Cierto número de autores (Hurtado, 2014; Poornachandrika, 2015; Abebe \& Markos, 2016; Valdivia, 2018) determinaron que existe una correlación positiva y significativa entre la SL y CO de los docentes universitarios. En el trabajo de investigación desarrollado, también se llegó al mismo resultado. 
Analizando la relación de la SL con las dimensiones del CO, Hurtado (2014) y Abebe \& Markos (2016) encontraron que la SL tiene correlación positiva y significativa con el compromiso afectivo y normativo, mas no así con el compromiso de continuidad. Por su parte, Valdivia (2018), determinó que la SL tiene relación positiva y significativa con las tres dimensiones (afectivo, de continuidad y normativo) del CO.

En este trabajo de investigación, controlando la presencia de variables confusoras mediante el uso de la regresión logística multivariada y tratando los datos según su escala de medición natural, se encontró que la SL tiene relación positiva y significativa sólo con el compromiso afectivo, mas no con el compromiso de continuidad ni con el compromiso normativo.

Respecto a la SL, Poornachandrika (2015) indica que el 85\% de los docentes tenián un nivel de SL bajo o promedio. Contrariamente, Valdivia (2018) determinó que sólo el 38.9\% de docentes tenían un nivel de SL bajo o medio. No obstante, en este estudio se ha determinado que, aproximadamente, la mitad de los docentes (48.3\%) se encontraban dissatisfechos laboralmente, lo cual no concuerda con los resultados de Poornachandrika (2015) y (Valdivia, 2018). Esta situación puede deberse a los diferentes intrumentos utilizados por los autores medir este constructo.

Respecto al CO, Poornachandrika (2015) y Valdivia (2018) indican que más de la mitad de docentes estudiados, tenián nivel de CO medio o alto, lo cual concuerda con el resultado encontrado en el trabajo realizado.

Una de las limitaciones en el desarrollo del trabajo fue la forma de obtención de los datos, puesto que los docentes indicaron tener "su tiempo muy ajustado", y por ello pocos se decidieron a participar con responsabilidad en las encuestas aplicadas. Por otra parte, para la obtención de datos sobre SL, se utilizó sólo el cuestionario adaptado de la adaptación de Academic Job Satisfaction Questionnaire (Al-Rubaish, et al., 2011).

Debido a la importancia del conocimiento de la SL y el CO de los docentes del sistema universitario peruano, a fin de lograr una educación universitaria de calidad y el bienestar de la sociedad, se sugiere la continuidad en la investigacion de estos temas; tomando los datos para la SL mediante el uso de los distintos intrumentos disponibles para este propósito. De igual manera, eligiendo los participantes en el estudio mediante el muestreo probabilístico y analizando los datos con técnicas de la estadística multivariada.

\section{Conclusión}


En el presente estudio se ha demostrado que existe relación directa y estadísticamente significativa entre la satisfacción laboral y el compromiso organizacional de los docentes de una universidad pública de Perú. Los docentes satisfechos laboralmente presentan 3.39 veces mayor la posibilidad de tener compromiso organizacional con la universidad, comparado con los docentes dissatisfechos; manteniendo controladas las posibles variables confusoras.

Por su parte, la satisfacción laboral no tiene relación con el compromiso de continuidad ni con el compromiso normativo; sin embargo, tiene relación directa y significativa con el compromiso afectivo; es así que los docentes satisfechos laboralmente, presentan 4.76 veces mayor la posibilidad de tener compromiso afectivo con la universidad, comparado con los docentes dissatisfechos; manteniendo controladas las posibles variables confusoras.

Por otro lado, el reconocimiento, las oportunidades de crecimiento y promoción de los docentes, que según la "Teoría de Herzberg”, son factores intrínsecos o motivacionales, no se encuentran en sus niveles óptimos en la universidad pública de estudio, ello no permite alcanzar altos niveles de satisfacción de los docentes.

En otro orden, el pago o remuneraciones de los docentes, la relación con los compañeros de trabajo y el entorno físico y comodidades que corresponden a los factores extrínsecos o higiénicos, según la "Teoría de Herzberg", no se encuentran en sus niveles óptimos en la universidad de estudio, ello conduce a la insatisfacción de sus docentes.

Desde otra parte, el alto porcentaje de dissatisfacción laboral, según versión de los docentes, se debe a los bajos niveles de los factores intrínsecos y extrínsecos de la "Teoría de Herzberg".

Finalmente, la mayoría de los docentes no tienen compromiso de continuidad en la universidad, principalmente debido al bajo nivel remunerativo que perciben comparado con alto costo de vida en la región, así también por la falta de oportunidades de crecimiento y reconocimiento.

\section{Referencias}

Abebe, T., \& Markos, S. (2016). The relathionsip between job satisfaction and organizational commitment in public higher education institute the case of Arba Minch University, Ethiopia. International Journal of Research in Business Management, 4(8), 17-36.

Al-Rubaish , A. M., Rahim, S.-I. A., Abumadini, M., \& Wosornu, L. (2011). Academic job satisfaction questionnaire: Construction and validation in Saudi Arabia. Journal of Family and Community Medicine, 18(1), 1. doi:10.4103/1319-1683.78630. 
Álvarez, C. H. (2019). Tendencias de diseños metodológicos en las publicaciones indexadas sobre la satisfacción laboral del profesorado universitario. Revista Electrónica Educare, 23(3), 1-23.

Alvarez-Gayou Jurgenson, J. L. (2003). Cómo hacer investigación cualitativa: fundamentos y metodología. México DF, México: Paidós Educador.

Barber, M., \& Mourshed, M. (2007). Cómo hicieron los sistemas educafivos con major desempeño del mundoI para alcanzar sus objetivos. Santiago de Chile, Chile: Informe McKinsey.

Barraza Macías, A. (2007). Apuntes sobre metodología de la investigación. La consulta a expertos como estrategia para la recolección de evidencias de validez basadas en el contenido. INED, 7, 5-14.

Casella, G., \& Berger, G. L. (1990). Statistical inference. California, EE.UU.: Duxbury Press.

Creswell, J. W. (2014). Research design: Qualitative, quantitative, and mixed methods approaches. California, EE.UU.: Sage Publications.

Dingeta,, M. G. (2013). Job Satisfaction and Organizational Commitment of Teacher Educators: The Case of Arbaminch College of Teacher Education (AMCTE). Addis Adaba.

Getahun, T., Tefera, B. F., \& Burichew, A. H. (2016). Teacher's Job Satisfaction And Its Relationship With Organizational Commitment In Ethiopian Primary Schools: Focus On Primary Schools Of Bonga Town. European Scientific Journal, 12(13).

Henquin, R. P. (2013). Epidemiología y Estadística para principiantes. Buenos Aires, Argentina: Corpus Libros Médicos y Científicos.

Hurtado Morales, L. K. (2014). Relación entre la percepción de justicia organizacional, satisfacción laboral y compromiso organizacional de docentes de una universidad privada de Bogotá. Tesis de grado, Universidad Catolica de Colombia. Bogotá. Colombia.

Liu, Y., \& Bellibas, M. S. (2018). School factors that are related to school principals' job satisfaction and organizational commitment. International Journal of Educational Research, 90, 1-19.

Long, J. S., \& Jeremy Freese . (2001). Regression Models for Categorical Outcomes Using Stata. Texas, EE.UU.: Stata Corporation.

Luna, M. (2015). Satisfacción laboral y compromiso organizacional del personal docente de una universidad privada de Lima. Tesis de maestría, Universidad Inca Garcilazo de la Vega, Lima, Perú. Recuperado de 
http://168.121.45.184/bitstream/handle/20.500.11818/385/Caratularesumen385.pdf?se quence $=4 \&$ is Allowed $=\mathrm{y}$

Maldonado-Radillo, S. E., Barón, M. R., Rivera, B. G., \& Venegas, A. C. (2014). Compromiso organizacional de los profesores de una universidad pública. Conciencia tecnológica, 47, 12-18.

Mena, T. M. (2016). Satisfacción laboral y copromiso organizacional en docentes de una universidad pública del Perú. Tesis de licenciatura, Universidad Señor de Sipán, Chiclayo, Perú. Obtenido de http://repositorio.uss.edu.pe/bitstream/handle/uss/3355/MENA\%20NEVADO\%20TA TIANA\%20MARCELA.pdf?sequence=1\&isAllowed=y

Meyer, J. P., \& Allen, N. J. (1997). Commitment in the workplace. California, EE.UU.: Sage Publications.

Monje Álvarez, C. A. (2011). Metodología de la investigación cuantitativa y cualitativa. Guía didáctica. Neiva, Colombia: Universidad surcolombiana.

Muhammad Hammad Ali, \& Imran Bashir. (2018). The effect of job satisfaction on teacher's organizational commitment, with special reference to private sector universities of Punjab, Pakistan. International Journal of Scientific and Research Publications (IJSRP), 8(2), 114-125. doi:http://dx.doi.org/10.29322/IJSRP.8.3.2018.p7519

Nieto, E. E. (2017). Análisis de la satisfacción laboral y su relación con el compromiso organizacional de los colaboradores del Instituto Tecnológico Superior Central Técnico, en el año 2017. Tesis de licenciatura, Pontificia Universidad Católica de Ecuador, Quito, Ecuador.

Poornachandrika, T. S. (2015). A Study of the Relationship between Job Satisfaction and Organizational Commitment of University Teachers in the Twin Citiies of Hyderabad and Secunderabad. International Journal of Engineering Technology, Management and Applied Sciences, 3.

Ramos, H., \& Santa Cruz, F. (2018). Satisfacción Laboral y Compromiso Organizacional de los Docentes del Instituto de Educación Superior Tecnológico Público del Distrito de Chalhuahuacho, Provincia de Cotabambas, Región Apurimac. Tesis de maestría, Universidad César Vallejo, Cusco, Perú.

Robbins, S. P., \& Coulter, M. (2012). Management. Boston, EE.UU.: Prentice Hall.

Rodríguez , A., \& Pérez, A. (2017). Métodos científicos de indagación y de construcción del conocimiento. Revista EAN, (82),179-200. 
Siegel, B., \& Castellan, N. J. (1998). Estadística no paramétrica aplicada a las ciencias de la conducta (4 ed.). Culiacán, México: Trillas.

Tentama, F., \& Pranungsari, D. (2016). The Roles of Teachers' Work Motivation and Teachers' Job Satisfaction in the Organizational Commitment in Extraordinary Schools. International Journal of Evaluation and Research in Education, 5(1), 39-45.

Valdivia, L. M. (2018). La satisfacción laboral y el compromiso organizacional de los docentes de la Facultad de Ecoturismo de la Universidad Nacional Amazónica de Madre de Dios, 2018. Tesis de maestría, Universidad César Vallejo, Puerto Maldonado, Perú. Recuperado de http://repositorio.ucv.edu.pe/bitstream/handle/UCV/27610/valdivia_ml.pdf?sequence= $1 \&$ isAllowed=y

Vargas, X. (2011). ¿Cómo hacer investigación cualitativa? Jalisco, México: ETXETA.

Werang, B. R., \& Agung, A. G. (2017). Teachers' job satisfaction, organizational commitment, and performance in Indonesia: A Study from Merauke District, Papua. International Journal of Development and Sustainability, 6(8), 700-711. 